\title{
Are naked singularities forbidden by the second law of thermodynamics?
}

\author{
Sukratu Barve and T. P. Singh \\ Theoretical Astrophysics Group \\ Tata Institute of Fundamental Research \\ Homi Bhabha Road, Bombay 400 005, India
}

\begin{abstract}
By now, many examples of naked singularities in classical general relativity are known. It may however be that a physical principle over and above the general theory prevents the occurrence of such singularities in nature. Assuming the validity of the Weyl curvature hypothesis, we propose that naked singularities are forbidden by the second law of thermodynamics.
\end{abstract}


The cosmic censorship hypothesis remains one of the most important unsolved problems in classical general relativity. Put in physical, non-rigorous, terms the hypothesis states that generic singularities arising in gravitational collapse are not visible. This hypothesis is crucial for the validity of blackhole physics and astrophysics. For instance, important theorems like the area-increase theorem for black-holes assume the validity of this hypothesis. The violation of the hypothesis implies that visible singularities can arise in gravitational collapse - these are called naked singularities.

Over the years, attempts to provide a proof of the censorship hypothesis have not been successful. On the other hand, many examples of naked singularities have been found in models of gravitational collapse in general relativity. Most of these examples have been found in studies of spherical collapse. These include (i) naked singularities in the collapse of inhomogeneous dust described by the Tolman-Bondi spacetime [1], (ii) naked singularities in the collapse of null dust described by the Vaidya spacetime [2], (iii) naked singularities in the collapse of perfect fluids [3], (iv) naked singularity at the critical point in the collapse of a massless scalar field [4]. There is also some evidence (though not incontrovertible) of naked singularity formation in the non-spherical collapse of dust [5]. While these examples are not sufficiently generic as to already invalidate the censorship hypothesis, their increasing number seriously suggests the possibility that the hypothesis may not be true after all. If so, black holes and naked singularities must be considered at par, from the point of view of classical general relativity.

The occurrence of a naked singularity represents the breakdown of predictability, because completely arbitrary data could be received by an observer who is to the future of the singularity. One might be compelled to consider modifying general relativity, so as to avoid naked singularity formation, and hence preserve predictability. However, as we discuss below, there appears to be a less drastic way out, which is consistent with presently known laws of physics, and does not require modification of the theory.

While general relativity does admit dynamical solutions leading to naked singularities, we should ask if these solutions are realized in the real physical world. Could it be that there is a physical principle, over and above general relativity, which forbids their occurrence in nature? For comparison, we recall the well-known advanced wave solutions of Maxwell's electrodynamics, whose occurrence in the real world is forbidden by the second law of 
thermodynamics. In a similar spirit, we propose in this essay that it is the second law which prevents the occurrence of naked singularities in nature. Naked singularities are seen to be analogous to the advanced wave solutions in electromagnetism - allowed by the theory, but not observed in the real world.

Our proposal is intimately connected with the Weyl curvature hypothesis put forward by Penrose [6]. As emphasized by Penrose, the entropy of the universe at its beginning must have been extremely low, in order for there to be something like the second law. There is very good evidence, however, that matter itself was in a high entropy, thermal equilibrium state. Thus matter by itself could not have been responsible for the overall low entropy. Hence the low entropy constraint at the Big Bang must come from the spacetime geometry - a suitably defined gravitational entropy must have had a remarkably low value. It is highly plausible that it is the Weyl curvature tensor which is a measure of gravitational entropy. This is because an initially Friedmannean universe will have zero Weyl curvature (and hence zero gravitational entropy). As the universe evolves, irregularities and clumping in the matter distribution develop; these result in a continual increase of the Weyl curvature and of gravitational entropy. Hence the hypothesis is stated as: the Weyl curvature of the universe is zero at the initial singularity. The second law of thermodynamics is then to be understood as a consequence of this hypothesis.

If cosmic censorship is assumed to be valid, then the final singularities, which are a result of gravitational collapse, will be black-holes. These singularities will have resulted from ever-increasing gravitational clumping, and typically the Weyl tensor will diverge in the approach to the black-hole singularity. Hence the structure of the final singularity will be greatly different from that of the initial singularity.

Our interest is in the implications of the Weyl curvature hypothesis if cosmic censorship fails. If a naked singularity results as the end state of gravitational collapse, it classifies as an initial singularity, because there are outgoing geodesics which terminate in their past at the singularity. The validity of the Weyl hypothesis requires that as the naked singularity is approached in the past along an outgoing geodesic, the Weyl curvature should go to zero [7]. This is essential because if general relativity admits a naked singularity solution in collapse, such a solution could in principle be indistinguishable from an initial cosmological singularity, for which case the hypothesis requires 
Weyl to be exactly zero.

Since a few analytical examples of naked singularities are known, it is easily possible to calculate the growth of the Weyl curvature along outgoing geodesics, as the singularity is approached in the past. Hence one could check for the validity of the hypothesis. For definiteness, we assume that it is the Weyl scalar $C \equiv C^{a b c d} C_{a b c d}$ which has to be zero at the initial singularity. Here we examine two models of spherical collapse which give rise to naked singularities. One is the spherical gravitational collapse of inhomogeneous dust, which is described by the Tolman-Bondi spacetime, for which the metric in comoving coordinates $(t, r, \theta, \phi)$ is

$$
d s^{2}=d t^{2}-\frac{R^{\prime 2}}{1+f(r)} d r^{2}-R^{2} d \Omega^{2}
$$

Here, $R(t, r)$ is the area radius at time $t$ of a shell with comoving coordinate $r$, and $f(r)$ is a free function which is determined by the initial density and velocity distribution of the dust cloud. The Weyl scalar $C$ is equal to

$$
C(t, r)=\frac{48}{R^{4}}\left(\frac{m(r)}{R}-\frac{m^{\prime}(r)}{3 R^{\prime}}\right)^{2}
$$

where $m(r)$ is the mass of the cloud interior to $r$. Note that $m(r)$ is time independent, (since we are dealing with dust) - it is determined by the initial density distribution. This scalar remains zero at the origin throughout the nonsingular phase of the evolution, but blows up at the curvature singularity, where the Kretschmann scalar also diverges.

It is known [1] that for certain initial density distributions, the singularity at $R=0, r=0$ resulting in dust collapse is at least locally naked, and there is a family of null geodesics emanating from the singularity. We define the tangent along an outgoing geodesic to be $X \equiv R / r^{\alpha}$ where $\alpha$ is a constant greater than unity, chosen so that the tangent is well-defined [1]. It can be shown that the limiting value of the Weyl scalar as the naked singularity is approached in the past along an outgoing geodesic is

$$
C\left(X_{0}, r\right)=\frac{16 \rho_{0}^{2} \theta_{0}^{2}}{3 X_{0}^{6}\left(X_{0}^{3 / 2}+\theta_{0}\right)^{2}} r^{6(1-\alpha)}
$$

Here, $\rho_{0}$ is the initial central density, $X_{0}$ is the limiting value of the tangent, and $\theta_{0}$ is a finite quantity determined by the initial density and velocity profile 
in the neighborhood of the origin. As we easily see by letting $r \rightarrow 0$, the Weyl scalar diverges at the naked singularity, along an outgoing geodesic. The same is true along an ingoing geodesic. We note that the divergence of the Weyl scalar and the occurrence of the naked singularity are both a consequence of the inhomogeneity of the initial density distribution. In a homogeneous collapse (the Oppenheimer-Snyder solution) the Weyl is exactly zero inside the cloud, and the collapse results in the formation of a black-hole. Also, the scalar is calculated using the Tolman-Bondi metric as the outgoing rays initially travel through the dust cloud.

As a second example, we consider the self-similar collapse of null dust. This is described by the Vaidya spacetime, which has the metric

$$
d s^{2}=\left(1-\frac{2 m(v)}{r}\right) d v^{2}-d r^{2}-r^{2} d \Omega^{2}
$$

Here $v$ is the advanced time coordinate, and $m(v)$ the mass function. For selfsimilar collapse, $m(v)=\lambda_{0} v, \lambda_{0}$ being a constant. This constant represents the rate of infall, $d m(v) / d v$, of the null dust. It is known that the singularity at $r=0, v=0$ resulting in collapse is naked for $\lambda_{0} \leq 1 / 8$ [2]. The Weyl scalar $C(v, r)$ is equal to $48 \mathrm{~m}^{2} / r^{6}$. The tangent to an outgoing geodesic which meets the naked singularity in the past is defined as $X=v / r$. Thus the Weyl scalar diverges as $\lambda_{0}^{2} X_{0}^{2} / r^{4}$ along the outgoing geodesic from the naked singularity, $X_{0}$ being the limiting value of the tangent. Once again the divergence of the Weyl and the occurrence of the naked singularity are both a consequence of the inhomogeneity of the spacetime.

We see that in both examples, the Weyl scalar diverges as the naked singularity is approached in the past. This is contrary to the expectations of the Weyl curvature hypothesis. In fact, since naked singularities in general arise when the matter distribution is highly inhomogeneous, one should actually expect the Weyl to diverge along outgoing geodesics, instead of going to zero. Hence the occurrence of naked singularities in collapse corresponds to initial singularities of very high Weyl curvature, and equivalently, very high gravitational entropy.

In order for the second law to hold, such initial singularities must be disallowed. Thus we are presented with two possibilities: (i) The Weyl curvature hypothesis and the suggested relation between Weyl and gravitational entropy is not true, and naked singularities occur in the real world. Or, alter- 
natively, (ii) the Weyl hypothesis is true, and as a consequence, the second law forbids the occurrence of naked singularities, because these represent initial configurations of very high entropy.

Which of these two alternatives is more likely to be the correct one? In our view, the Weyl hypothesis is an important and perhaps the unique step towards an understanding of the second law. Hence it should not be sacrificed. On the other hand, naked singularities are predicted by general relativity, but apparently not observed in the real world. Hence we propose that it is thermodynamics, via the Weyl curvature hypothesis, which forbids the occurrence of naked singularities in nature. This would imply that somehow nature remarkably avoids those initial conditions in stellar collapse which eventually lead to naked singularities.

\section{References}

[1] see for instance, T. P. Singh and P. S. Joshi, Class. Quantum Grav. 13 (1996) 559, and references therein.

[2] see, e.g., W. A. Hiscock, L. G. Williams and D. M. Eardley, Phys. Rev. D 26 (1982) 751.

[3] A. Ori and T. Piran, Phys. Rev. D 42 (1990) 1068; P. S. Joshi and I. H. Dwivedi, Commun. Math. Phys. 146 (1992) 333.

[4] M. W. Choptuik, Phys. Rev. Lett. 70 (1993) 9.

[5] S. L. Shapiro and S. A. Teukolsky, Phys. Rev. Lett. 66 (1991) 994.

[6] R. Penrose in General Relativity, Eds. S. W. Hawking and W. Israel, Cambridge University Press (1979).

[7] R. Penrose in Quantum Gravity 2, Eds. C. J. Isham, R. Penrose and D. W. Sciama, Oxford (1981). 